International Journal of Pure and Applied Mathematics

Volume 105 No. 4 2015, 795-804

ISSN: $1311-8080$ (printed version); ISSN: 1314-3395 (on-line version)

url: http://www.ijpam.eu

doi: http://dx.doi.org/10.12732/ijpam.v105i4.18

ijpam.eu

\title{
A NOTE ABOUT FREQUENCIES BEING CLOSE OR AMPLITUDES BEING SMALL IN FREQUENCY ANALYSIS
}

\author{
Ragnhild Johanne Rensaa \\ Narvik University College \\ P.O.Box 385, N-8505 Narvik, NORWAY
}

\begin{abstract}
In an earlier paper, an alternative method for how to solve the frequency analysis problem was offered. The object of the present paper is to bring about how to handle situations where the frequencies are close to each other or there is an amplitude that is really small.
\end{abstract}

AMS Subject Classification: 30C45

Key Words: frequency analysis, univalent functions, close frequencies, small amplitudes

\section{Processes to Identify Frequencies}

\subsection{A Well-Known Way of Identifying Frequencies}

The frequency analysis problem is the problem of determining the unknown frequencies $\omega_{j}$ and amplitudes $\alpha_{j}$ in a trigonometric signal. A method for solving the problem may roughly be described as follows: Based on signal values, a certain absolutely continuous measure on the unit circle is constructed. Next, an inner product is defined and in turn moments and monic orthogonal polynomials are obtained. Finally, asymptotic values of some zeros of the polynomials lead to the frequencies. (See [2], [3], [4], [5], [6], [8] for details). More precisely,

Received: October 10, 2015

(c) 2015 Academic Publications, Ltd. url: www.acadpubl.eu 
a given trigonometric signal may assumed to be on the form

$$
G(t)=\sum_{j=-I}^{I} A_{j} e^{2 \pi i f_{j} t},
$$

where $t$ is the time, $f_{j}=-f_{-j}, j=1,2, \ldots, I, f_{0}=0$, the frequencies and $A_{j}=A_{-j} \in \mathbb{R},\left|A_{j}\right|$ the amplitudes. The frequency analysis problem is to find the unknown frequencies from signal values observed at times $m \Delta t$. With $\omega_{j}=2 \pi f_{j} \Delta t$ arranged so that $0<\omega_{1}<\omega_{2}<\cdots<\omega_{I}<\pi$, the signal may then be written on the form

$$
x(m)=2 \sum_{j=1}^{I} A_{j} \cos m \omega_{j} .
$$

Since $+\omega_{j}$ and $-\omega_{j}$ represent the same frequency, we count them as one and let $I$ denote the number of frequencies. Let for any positive integer $N, \psi^{(N)}(\theta)$ be the positive measure on the unit circle $\partial \mathbb{D}=\{z:|z|=1\}$ given by

$$
\frac{d \psi^{(N)}(\theta)}{d \theta}=\frac{1}{2 \pi}\left|\sum_{m=0}^{N-1} x(m) e^{-m i \theta}\right|^{2} .
$$

This measure defines $\left\{\mu_{n}^{(N)}\right\}_{-\infty}^{\infty}$; a sequence of moments

$$
\mu_{n}^{(N)}=\int_{-\pi}^{\pi} e^{-n i \theta} d \psi^{(N)}(\theta), \quad n=0, \pm 1, \pm 2, \ldots,
$$

and gives rise to an inner product and a sequence of monic orthogonal polynomials $\rho_{n}^{(N)}(z)$ called Szegö polynomials. The Szegö polynomials may be determined in different ways: 1: By determinant formulas, 2: By the Levinson algorithm, 3: By a method in which

$$
L_{0}(z):=\mu_{0}^{(N)}+2 \sum_{k=1}^{\infty} \mu_{k}^{(N)} z^{k}, \quad \mu_{k}^{(N)} \text { as in (1), }
$$

represents a Carathéodory function, mapping the unit disk into the open right halfplane $\Re w>0 . L_{0}$ plays an essential role in the alternative method for frequency identification introduced in Section 1.2. Together with the power series

$$
L_{\infty}(z):=-\mu_{0}^{(N)}-2 \sum_{k=1}^{\infty} \mu_{-k}^{(N)} z^{-k}
$$


which represents an analytic function in $|z|>1$ mapping it into $\Re w<0, L_{0}$ correspond to a unique positive continued fraction expansion, called a PerronCarathéodory fraction:

$$
\delta_{0}-\frac{2 \delta_{0}}{1}+\frac{1}{\overline{\delta_{1}} z}+\frac{\left(1-\left|\delta_{1}\right|^{2}\right) z}{\delta_{1}}+\frac{1}{\overline{\delta_{2}} z}+\frac{\left(1-\left|\delta_{2}\right|^{2}\right) z}{\delta_{2}}+\cdots
$$

The Szegö polynomials are denominators of the odd order approximants of this particular continued fraction, when it is constructed in a certain way from the signal. For more details, see [1], [7].

In the sequence $\left\{\mu_{n}^{(N)}\right\}$ we make two replacements: First, all formulas remain valid if all $\mu_{n}^{(N)}$ are replaced with $\mu_{n}^{(N)} / N$. By the convergence of $\psi^{(N)}(\theta) / N$ it then follows that

$$
\lim _{N \rightarrow \infty} \mu_{n}^{(N)} / N=: \mu_{n}=\sum_{j=-I}^{I} A_{j}^{2} e^{n i \omega_{j}}=A_{0}^{2}+2 \sum_{j=1}^{I} A_{j}^{2} \cos n \omega_{j} .
$$

We replace $\left\{\mu_{n}\right\}$ by $\left\{\mu_{n} R^{|n|}\right\}$ for any fixed $R \in(0,1)$. Using $\mu_{n} R^{|n|}$ instead of $\mu_{n}^{(N)} / N$ in the determinant formula for Szegö polynomials, the limit as $R \rightarrow 1$ exists for any $n$. For $n>n_{0}=2 I+L$ where $L=1$ for $A_{0} \neq 0$ else $L=0$, the limit polynomial will contain

$$
(z-1)^{L} \prod_{j=-I}^{I}\left(z-e^{i \omega_{j}}\right)\left(z-e^{-i \omega_{j}}\right)
$$

as factor. This R-process leads to the unknown frequencies without going through subsequences.

\subsection{An Alternative Way of Identifying Frequencies}

In an earlier paper [9], an alternative method for how to solve the frequency analysis problem is presented. We use the fact that for a normalized starlike univalent function

$$
f(z)=z+a_{2} z^{2}+\cdots+a_{n} z^{n}+\cdots, \quad z \in \mathbb{D},
$$

the expression $\frac{z f^{\prime}(z)}{f(z)}$ is a Carathéodory function. The idea is then to solve the differential equation

$$
\frac{z f^{(N) \prime}(z)}{f^{(N)}(z)}=L_{0}(z)
$$


In [9] it is proved that

$$
\lim _{N \rightarrow \infty} f^{(N)}(z)=: f(z)=\frac{z}{\prod_{j=1}^{I}\left(1-2 z \cos \omega_{j}+z^{2}\right)^{2 \alpha_{j}^{2}}} \in S^{*}
$$

where

$$
\alpha_{j}=\frac{A_{j}}{K}, K=\sqrt{2 \sum_{j=1}^{I} A_{j}^{2}} \text { to give } 2 \sum_{j=1}^{I} \alpha_{j}^{2}=1 .
$$

The function $f$ is called a slit mapping function. By analyzing the picture of $f(\mathbb{D})$ it is shown that:

1. The number of frequencies of the signal is the number of angular openings in the upper half plane.

2. The absolute values of the amplitudes of the signal are the square root of the angles between the rays divided by $2 \pi$.

3. The frequencies of the signal are determined by either:

- using the exact number of frequencies from point 1. in the Szegö polynomial method, or

- solving a set of signal equations using known values of the signal, or

- solving a set of selected signal equations from the previous point and in addition some moment equations using known values of the moments, or

- measuring the distances to the slit endpoints and use the formulas for the distances.

See [9] for details.

For practical purposes, we can only include a finite number of terms in the Taylor expansion of (3). This, together with the need to approximate the unit disk to a slightly smaller one, produce errors in our calculations. These errors are analysed and estimated influence of in [10].

Example 1. (Slit Mapping Illustrations)

Let $I=3$ with $\omega_{1}=\frac{\pi}{8}, \omega_{2}=\frac{\pi}{3}, \omega_{3}=\frac{3 \pi}{4}$ and $2 \alpha_{1}^{2}=\frac{1}{6}, 2 \alpha_{2}^{2}=\frac{1}{2}, 2 \alpha_{3}^{2}=\frac{1}{3}$. Then (3) takes the form

$$
f(z)=\frac{z}{\left(1-2 \cos \left(\frac{\pi}{8}\right) z+z^{2}\right)^{\frac{1}{6}}\left(1-z+z^{2}\right)^{\frac{1}{2}}\left(1+\sqrt{2} z+z^{2}\right)^{\frac{1}{3}}} .
$$




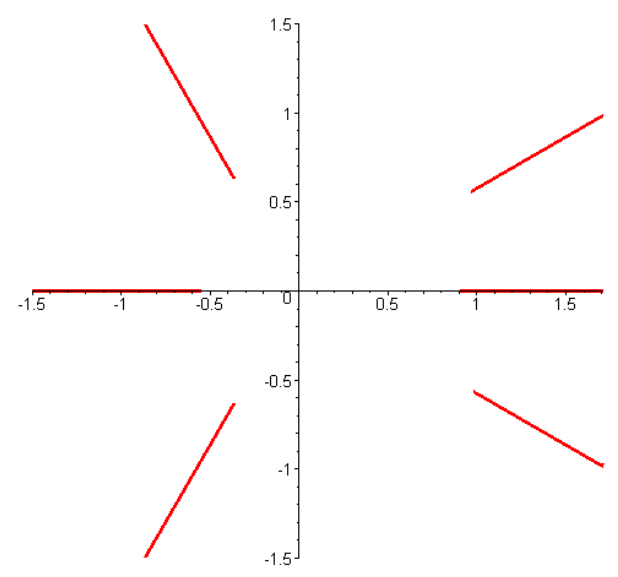

Figure 1a

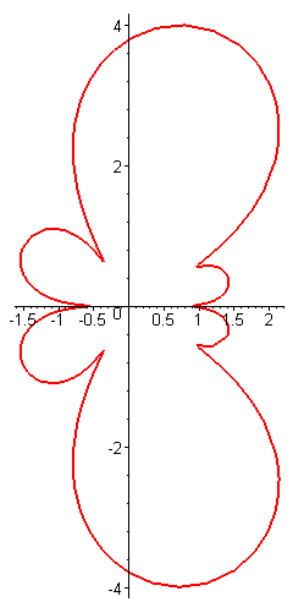

Figure 1b

This function maps $\mathbb{D}$ onto the complement of two times three slits, see Figure 1a. In a practical case we have to map $|z|<R<1$ or by the maximum principle $|z|=R$. This produces a domain like in Figure 1b, made by using Maple as working tool. The slit endpoints look like cusps on the illustration, but angular openings are still possible to determine and distances to the slit endpoints are pretty much the same.

To determine the number of frequencies, we depend upon the possibility to count the number of angular openings in the upper halfplane. In this process two errors may occur: Frequencies are close or one amplitude is much larger than another. We illustrate two situations like these in the following examples.

Example 2. (Close Frequencies)

To concentrate on close frequencies, we choose an example with $2 \alpha_{1}^{2}=$ $2 \alpha_{2}^{2}=\frac{1}{2}$. This produces slits on the real an imaginary axis. To show the difference between "close" and "very close" frequencies and only one frequency, we pick a: $\omega_{1}=\frac{\pi}{3}-\frac{3 \pi}{180}, \omega_{2}=\frac{\pi}{3}+\frac{3 \pi}{180}$ b: $\omega_{1}=\frac{\pi}{3}-\frac{\pi}{180}, \omega_{2}=\frac{\pi}{3}+\frac{\pi}{180}$ and c: $\omega_{1}=\omega_{2}=\frac{\pi}{3}$. The result is shown in Figure 2abc. In all these figures we have chosen $R=0.98$. As seen, when frequencies are close, only the "flatness" of the ball above the real axis can indicate the possibility of having more than one frequency.

If a situation like in Figure 2b occur and we suspect two close frequencies, it is possible to take a smaller interval around the value where the curve crosses the imaginary axis. Also, we may increase the radius of the circle $\partial \mathbb{D}_{R}:=\{z$ : $|z|=R\}$ to be mapped. In Figure $2 \mathrm{~d}$, we have chosen $R=0.995$ and restricted 


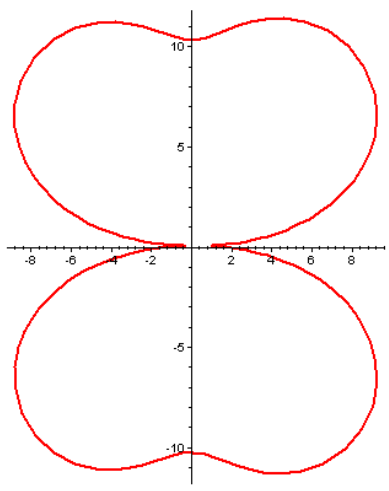

Figure 2a

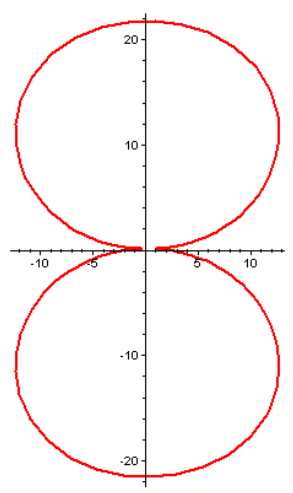

Figure 2a

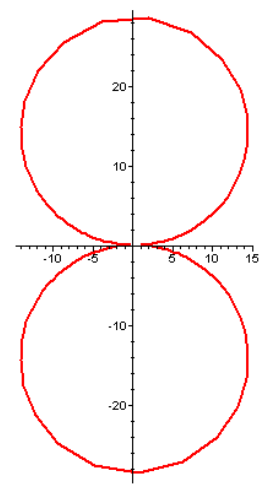

Figure 2c

the mapping interval. As seen, there is a slit on the imaginary axis. If we do the same for a situation like in Figure 2c, it will not give any change.

But there may be situations where we need other ways to chech whether or not we have adjacent frequencies. In the next section some observations are made towards how to deal with the problem in another way.

Example 3. (One Really Small Amplitude) If we have $2 \alpha_{1}^{2}=\frac{1}{3}, 2 \alpha_{2}^{2}=\frac{2}{3}$ and normalized frequencies $\omega_{1}=\frac{\pi}{6}, \omega_{2}=\frac{3 \pi}{4}$, it is not difficult to count the number of bumps to state the number of frequencies, see Figure 3a. But if we have $2 \alpha_{1}^{2}=\frac{1}{30}, 2 \alpha_{2}^{2}=\frac{29}{30}$ and unchanged frequencies, Maple produce a figure 


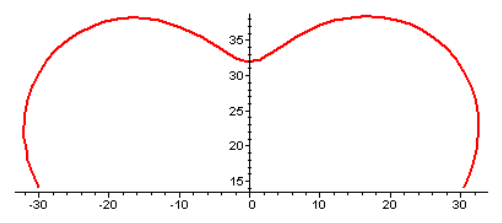

Figure 2d

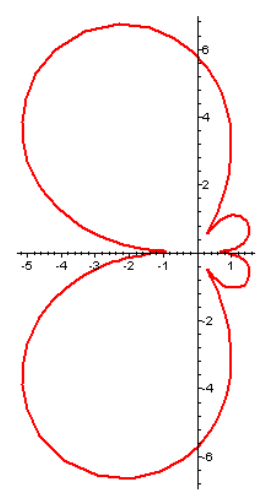

Figure $3 \mathrm{a}$

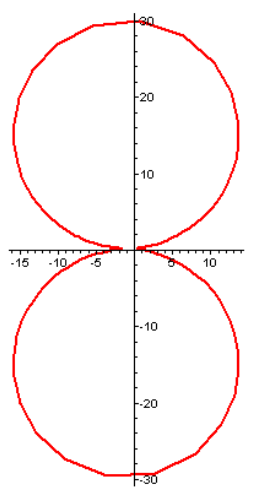

Figure $3 \mathrm{~b}$

like the one in Figure 3b. As seen, it is not possible to separate the bumps near $\mathbb{R}^{+}$since one of the amplitudes is so small.

But also in situation where we suspect small amplitudes, it is possible to incrase the mapping radius and restrict the mapping interval to investigate parts of the curve more closely. The adventage about small amplitudes is that they always occur close to an already existing slit endpoint, in our example near the positive real axis. Thus, it may be wise to chech around all slit endpoints anyway. 


\section{About Frequencies being Close and Amplitudes being Small}

Assume two different frequencies $\omega_{1} \neq \omega_{2}$. Without loss of generality we choose corresponding amplitudes to be $A_{1}=a / \sqrt{2}, A_{2}=1 / \sqrt{2}, a>0$. Then, use the $R$ - process to calculate the moments

$$
\mu_{n}=\left(a^{2} \cos n \omega_{1}+\cos n \omega_{2}\right) R^{n}, n=0,1, \ldots, 5 .
$$

To obtain nicer expressions, we use the Tchebyecheff formulas to rewrite all expressions in term of $\cos \omega_{1}$ and $\cos \omega_{2}$, and substitute $x=\cos \omega_{1}, y=$ $\cos \omega_{2}$. Then, the formula (5) produces manageable expressions for the moments $\mu_{0}, \ldots, \mu_{5}$. These moments are substituted into the determinant formula for the Szegö polynomial of degree 5 . The limit as $R \rightarrow 1$ is $\rho_{5}(z)$, in which our main interest is the constant term. Four of the zeros are the frequency points. The fifth one, being minus the constant term, is the uninteresting zero. The process leads to the following lemma:

Lemma 4. Given the signal

$$
x(m)=\sqrt{2}\left(a \cos m \omega_{1}+\cos m \omega_{2}\right) .
$$

Let $\rho_{5}(z)$ be the limit as $R \rightarrow 1$ of the Szegö polynomial of degree 5 in the $R$-process. Then the constant term in $\rho_{5}(z)$ is

$$
K_{a}(x, y)=\frac{4 x^{2} y+4 a^{2} x y^{2}+3 a^{2} x+8 x+8 a^{2} y+3 y}{4\left(2 x^{2}+2 a^{2} y^{2}+2 a^{2} x y+2 x y+1+a^{2}\right)},
$$

where $x=\cos \omega_{1}, y=\cos \omega_{2}$.

Proof. Simple, but non-trivial use of Maple.

From this Lemma, observations can be made both about adjacent frequencies and small amplitudes.

\subsection{A way to handle two close frequencies or one frequency}

Consider a situation where it is a possibility of having two adjacent frequencies. We assume $\omega_{1}=u-t, \omega_{2}=u+t$ for a $t>0$. In order to compare to the case where we only have one frequency, we let $t \rightarrow 0$ in (6), which means that $y \rightarrow x$. On the other hand, if we assume only one frequency meaning that $t=0$ from start, we have three uninteresting zeros and the constant term is minus the product of these. We obtain the following result: 
Proposition 5. The constant term of the Szegö polynomial $\rho_{5}(z)$ when $t \rightarrow 0$ is

$$
K_{a}(x)=\frac{4 a^{2} x^{3}+4 x^{3}+11 a^{2} x+11 x}{4\left(4 a^{2} x^{2}+4 x^{2}+1+a^{2}\right)},
$$

while the constant term when there is only one frequency is

$$
K(x)=-\frac{x^{3}}{4\left(x^{2}-2\right)} .
$$

As seen, we have an obvious discontinuity for $t=0$ since $t \rightarrow 0$ leads to (7) and $t=0$ leads to (8). This may be used to state if we have two close or only one frequency:

If we suspect close frequencies because of "flatness" in a slit mapping figure, the natural thing is to map an arc of $\partial \mathbb{D}_{R}$ to zoom in a smaller area on the figure. By that we may be able to count the number of frequencies. Alternatively, if the constant term of the Szegö polynomial $\rho_{5}(z)$ is given - for instance by a calculation of the PC-fraction in (2) - this value may be compared to (7) and (8) to conclude whether or not there is one or two frequencies.

Comment: Although the argument above is given in case of one frequency or two close ones, we believe that it can be generalized to a larger number of frequencies.

\subsection{A Way to Handle Small Amplitudes}

To consider what happens when we have one really small amplitude, a situation with only two frequencies will be the worst case. This is because the requirement $2 \sum_{j=1}^{I} \alpha_{j}^{2}=1$ in (4) will imply that the other amplitude is big. Let $\omega_{1}, \omega_{2}$ be two different but fixed frequencies and let $a \rightarrow 0$.

We have the following result:

Proposition 6. The constant term of the Szegö polynomial $\rho_{5}(z)$ when $a \rightarrow 0$ is

$$
K_{0}(x, y)=\frac{4 x^{2} y+8 x+3 y}{4\left(2 x^{2}+2 x y+1\right)} \text { when } s>0, s \rightarrow 0 .
$$

If, on the other hand, we assume $a=0$ from start, the constant term is of course the same as in (8).

Note. In the well-known method for frequency identification, a small amplitude is equally important to a large one: The method does not weight the small one less than the other. In the alternative method, however, the size of the amplitude decide its "role" in the complete signal. 


\section{References}

[1] W.B.Jones, O.Njåstad and W.J.Thron, Continued fractions associated with trigonometric and other strong moment problems Constr. Approx. 2 (1986), 197-211.

[2] W.B.Jones, O.Njåstad, W.J.Thron and H.Waadeland, Szegö polynomials applied to frequency analysis J. Comput. Appl. Math. 46 (1993), 217-228.

[3] W.B.Jones, O.Njåstad and H.Waadeland, An alternative way of using Szegö polynomials in frequency analysis, in S.C.Cooper and W.J.Thron eds. Cont. Frac. and Orth. Func., Marcel Dekker, New York, 141-152 (1994).

[4] W.B.Jones, V.Petersen Frequency analysis, Szegö polynomials and PCcontinued fractions Acta Applicandae Mathematicae 61 (2000), 149-174.

[5] O.Njåstad and H.Waadeland, General Szegö theory in frequency analysis, J. Math. An. and Appl. 206 (1997), 280-307.

[6] O.Njåstad and H.Waadeland, Asymptotic properties of zeros of orthogonal rational functions, J. Comput. Appl. Math. 77 (1997), 255-275.

[7] V.Petersen and H. Waadeland, Szegö polynomials in Frequency analysis, J. Comput. Appl. Math. 139 (2002), 239-251.

[8] K.Pan and E.B.Saff, Asymptotics for Zeros of Szegö polynomials associated with trigonometric polynomial signals, J. Approx. Theory 71 (1992), 239251.

[9] R.J.Rensaa, Univalent Functions and Frequency Analysis. Rocky Mountain Journal of Mathematics, Vol. 33, No. 2, (2003), 743-758.

[10] R.J.Rensaa, Estimated Errors in Univalent Functions and Frequency Analysis. International Journal of Pure and Applied Mathematics, Vol. 42, No. 1, (2008), 743-758. 